\title{
Comfortable Working Area of Seated Operators
}

\author{
Eui S. Jung* and Sungjoon Park** \\ * Department of Industrial Engineering, Pohang University of Science and Technology, \\ San 31 Hyoja, Pohang, 790-784, KOREA \\ ** Samsung Motors, Technology Center, 493, Banwol-Ri, Taean-Eup, Hwasung-Kun, \\ Kyungki-Do, 445-970, KOREA
}

\begin{abstract}
Comfortable working area for knob and lever controls was developed to modify the concept of conventional working area. A proposed working area based on the actual working comfort of an operator avoids dichotomy which is the reachability of control devices, and shows a distinct shape compared with existing normal working area following the Farley's concept. It is shown that the distance from the body to control devices and the slope of a work table have the quadratic effect to working comfort, and that the most comfortable area for the controls is located at the distance of about $2 \sim 4 \mathrm{~cm}$ in the sagittal direction and about $42 \sim 43 \mathrm{~cm}$ in the frontal direction from the shoulder respectively, which is located outside the existing normal working areas. The result of the response surface analysis indicates that a little slope of the control panel, about $15^{\circ}$, improves the working comfort. From the study, it was found that the working comfort varies within the working area even if each position is equally far from the body. It is suggested that the conventional normal working area needs to be refined based on the actual working comfort. Moreover, the comfortable working area could differ from control to control, and the primary working area should be determined by the type of a control. It is expected that the isocomfort working area generated in this study is used as a useful guideline for control panel layout.
\end{abstract}

Keyword : comfortable working area, psychophysical evaluation, working comfort

\section{INTRODUCTION}

For efficient operation, it is essential that vital hand controls must be easily reached and maneuvered by the operator from his or her normal working position. Workplace should be designed so that it will ensure greater productivity by maximizing work efficiency at a minimum human cost. Farley (1955) determined the normal working area in the horizontal plane simply by drawing an arc with a sweep of the right or left hand. Squires (1956) recognized that the arm does not move in a circular path and indicated that the path described by the hand when the forearm moved about a relaxed upper arm would be a prolate epicycloid. Squires' curve provided a substantial increase over Farley's normal working area.

These conventional concepts of the normal working area which are based on the anthropometry data and the movement of arms have certain problems although they are practically easy to apply due to their simplicity (Jung et al., 1995). First, traditional studies considered only the reachability at the specific posture, and equal performance was assumed within the same working area. However, the equal distances from the body center do not guarantee the same working comfort and it may yield quite a difference in operator's performance for prolonged work. Second, they used only the length and the trajectory of operator's arm, and no measure was employed in the determination of the working area. Finally, they provided the working area just for the horizontal workplace despite that many control panels and workplaces in industry have a proper slope for improving the working condition.

In designing the workplace, the primary controls should be positioned in a more comfortable area so as to increase the work performance and to decrease the working fatigue since operators work in eight hours or more in many cases. Therefore, the concept of comfortable working area is proposed in this study. The working comfort at each position within the working area was measured through the experiment that simulates the manipulation of a real control instrument. The objectives of this study are first to develop the functional relationship between working comfort and the position of equipments on a workplace, and second, to obtain isocomfort working area reflecting the effects of the factors such as the distance of a control instrument from the body center and the slope of the work table. Although the working comfort may be usefully used as a criteria for determining the primary working area, it is difficult to measure quantitatively. In this study, the working comfort for control instruments was measured by the magnitude estimation technique, a type of psychophysical evaluation methods. 


\section{Method}

\subsection{Subject and apparatus}

Four female and six male graduate and undergraduate students voluntarily participated in the experiment. Their statures ranged from 5th percentile of the female to 95th percentile of the male, with their ages ranged from 20 to 28. All subjects described themselves as right-handed. Two types of controls were used for the experiments measuring the working comfort. A lever was selected as a control device which is representative of gripping operation, and a knob of finger operations. They are also representative of linear and rotational operation, respectively. A first-order tracking task was given to the subject using an IBM-compatible PC. The subject was instructed to operate the control on a work table to track a continuously moving target in the computer screen.

2.2 Experimental Design

A mixed-factors design was used in this study. Factors investigated were "Sagittal Distance (SD)", "Frontal Distance (FD)", "Slope (S)", and "subject's Percentile by stature (P)". SD and FD are the sagittal and frontal distances from the shoulder to the position of the instrument, respectively, and $S$ indicates the slope of a work table in which the instrument is operated. Among these variables, SD, FD, and $\mathrm{S}$ are within-subjects variables, and $\mathrm{P}$ variable is a between-subjects variable. The dependent measure, perceived comfort for the control operation, was collected for each treatment condition.

Since the independent variables except for $P$ variable were expected to show no more than quadratic effect to the working comfort, the response surface methodology was employed to develop a prediction model for the working comfort with respect to the instrument location. The central-composite design was used to build up a response surface model. For the spherical central composite design, each factor appears at five distinct levels $(-\alpha$, $-1,0,+1,+\alpha)$. And, for the orthogonal central composite design, $\alpha$ value was set at 1.414 for four factors design with an equal replication in each treatment (Myers, 1976). Table 1 represents the five levels of four independent variables. For the sagittal distance, positive and negative signs indicate the right-side and left-side of the subject, respectively.

\subsection{Procedure}

Table 1. Factor levels of the central composite design

\begin{tabular}{|c|c|c|c|c|c|}
\hline Factor & $-\alpha$ & -1 & 0 & 1 & $+\alpha$ \\
\hline $\mathrm{SD}(\mathrm{cm})$ & -60 & -42 & 0 & 42 & 60 \\
\hline FD $(\mathrm{cm})$ & 0 & 9 & 30 & 51 & 60 \\
\hline (degree) & 0 & 4 & 15 & 26 & 30 \\
\hline$(\mathrm{cm})$ & 150 & 154 & 164 & 174 & 178 \\
\hline
\end{tabular}

The subject was asked to manipulate a control instrument according to the treatment condition and to express the magnitude of the perceived working comfort using numeric estimate. Upon completion of 15 treatment conditions, a psychophysical scaling task was conducted in which the subject was instructed to provide numeric estimates for 9-point verbal descriptors describing the degree of comfort ranging from "extremely poor" to "extremely good".

\section{Results}

A regression model, or more precisely, a response surface model was constructed between working comfort and four independent variables for each control. The model consists of three linear components (SD, FD, S), three quadratic components $\left(\mathrm{SD}^{2}, \mathrm{FD}^{2}, \mathrm{~S}^{2}\right.$ ), three linear-by-linear crossproduct components (SDxFD, SDxS, FDxS), and a covariate variable $(\mathrm{P})$. Some of these variables were found to be statistically insignificant in the model and to have little contribution to prediction precision. To select a new model which has better performance in terms of prediction variance and bias, $\mathrm{Cp}$ statistic, adjusted $\mathrm{R}^{2}$, and MSE were adopted as model selection criteria. The prediction models were built for each control type individually. The final prediction models selected by the three criteria are as follows;

$$
\begin{aligned}
& \text { Working Comfort }=76.2-5.76 x \mathrm{xD}-9.99 \mathrm{xFD}-25.6 \mathrm{xSD}^{2}-10.9 \mathrm{xDD}^{2}-5.2 \mathrm{xS}^{2}+2.9 \mathrm{xFDxS}+2.96 \mathrm{xP} \\
& \left(\mathrm{R}^{2}=0.71\right. \text {, Lever control, Right-hand) } \\
& \text { Working Comfort }=70.5-5.87 \mathrm{xSD}-8.62 \mathrm{xFD}-25.4 \mathrm{xSD}^{2}-10.5 \mathrm{xFD}^{2}-5.05 \mathrm{xS}^{2}+2.4 \mathrm{xFDxS}+2.17 \mathrm{xP} \\
& \left(\mathrm{R}^{2}=0.69\right. \text {, Lever control, Left-hand) } \\
& \text { Working Comfort }=71.5-3.59 \times \mathrm{xSD}-9.08 \mathrm{xFD}+1.81 \times \mathrm{SS}-22.6 \mathrm{xSD}^{2}-8.39 \mathrm{xFD}^{2}-3.51 \times \mathrm{xS}^{2} \\
& -2.06 \mathrm{xSDxFD}+1.89 \times \mathrm{xDxS}+4.62 \times \mathrm{P}, \quad\left(\mathrm{R}^{2}=0.74, \text { Knob control, Right-hand }\right) \\
& \text { Working Comfort }=66.0-4.11 x S D-9.48 x F D+1.86 x S-22.9 x \mathrm{xD}^{2}-8.15 \mathrm{xFD}^{2}-3.95 \mathrm{xS}^{2}+1.53 \mathrm{xP}
\end{aligned}
$$$$
\left(\mathrm{R}^{2}=0.70,\right. \text { Knob control, Left-hand) }
$$ 
From the lack of fit test, these models are appropriate to sufficiently explain the functional relationship between working comfort and the factors considered (all p-values were greater than 0.1 for the above models).

\section{Comfortable Working Areas}

Based on the response surface analysis which showed that linear and quadratic effects of independent variables were significant, the predicted comfort for two control operations were obtained for each position on a work table. The predicted comfort was plotted to generate an isocomfort working area, in which all the positions on a curve produced the same working comfort for each slope of a work table. Figures 1 and 2 show representative isocomfort working areas with respect to the control type, the percentile of the subject and the slopes of a work table. Isocomfort working area was plotted according to the verbal descriptor in three comfort levels such as "good". "good a little", and "so-so".

In this study, the comfortable working area of the right hand is larger than that of the left hand because the subjects are right-handed. The area was larger at the slope of $15^{\circ}$ than at the horizontal workplce for both controls. A little slope can, therefore, increase working comfort significantly.

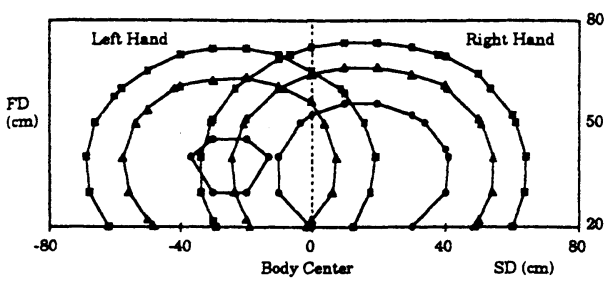

(a) 95 th percentile male at the slope of $0^{\circ}$

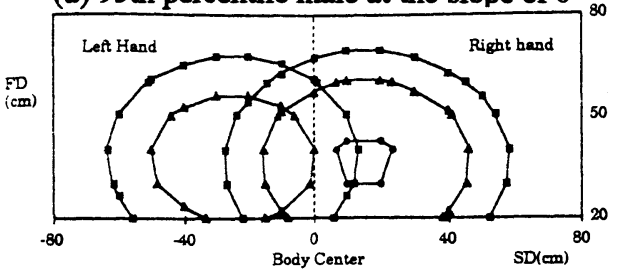

(c) 5th percentile female at the slope of $0^{\circ}$

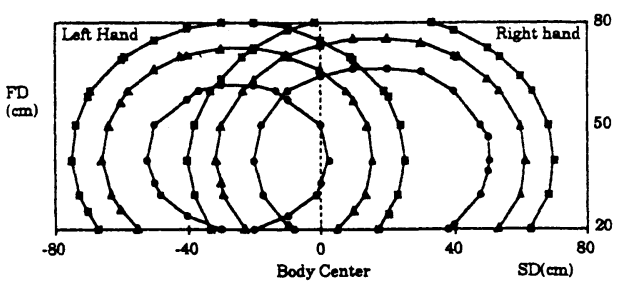

(b) 95 th percentile male at the slope of $15^{\circ}$

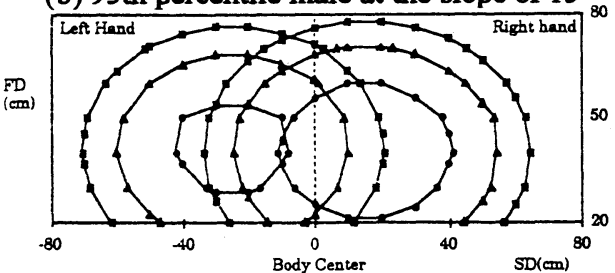

(d) 5th percentile female at the slope of $15^{\circ}$

$\longrightarrow$ good good a little $\longrightarrow$ s0 so

Figure 1. Isocomfort working areas for lever control

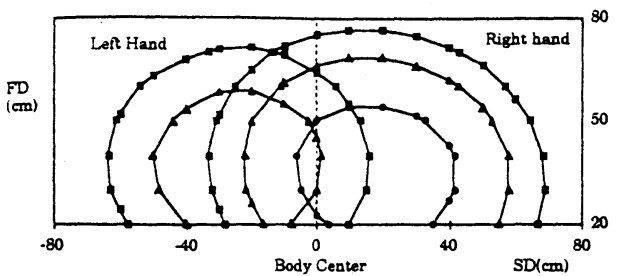

(a) 95 th percentile male at the slope of $0^{\circ}$

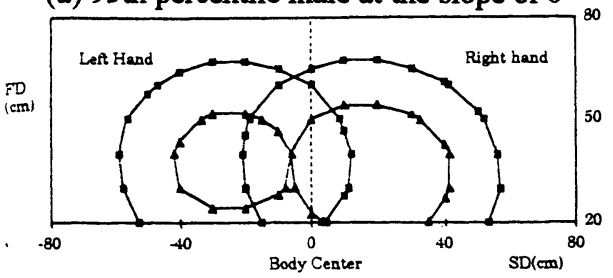

(c) Sth percentile female at the slope of $0^{\circ}$

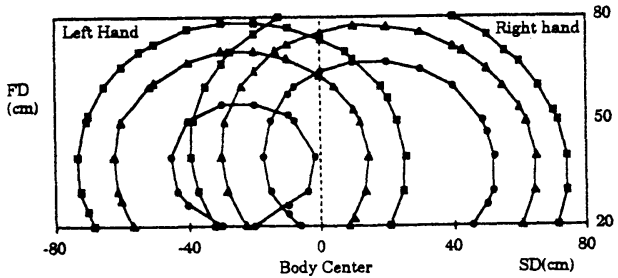

(b) 95th percentile male at the slope of $15^{\circ}$

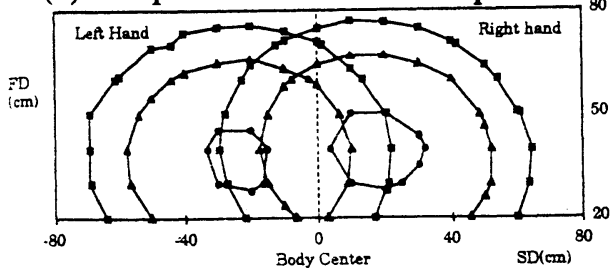

(d) 5th percentile female at the slope of $15^{\circ}$

Figure 2. Isocomfort working areas for knob control 


\section{Discussion and conclusions}

It is apparent from the contour of isocomfort working area that working comfort is quite different for each position although the positions are equally far from the body center. Working comfort can be different depending on the posture even if the distances to those instrument locations from the body are the same. It is, therefore, not appropriate to determine the primary working area based only on the distance from the body like Farley's normal working area. Particularly, a part of the most comfortable working area lies outside the normal working arae by Farley, as shown in Figure 3. It is thus suggested that the traditional curves of the normal working area need to be refined based on operator's comfort.

Figure 3 shows that working comfort at the location even outside the maximum working area by Farley can be better than the working comfort inside the normal working area. That is, the traditional concept of dichotomy which divides the working area as inside or outside the normal working area may be inappropriate in the design of a workplace or a control panel. The concept of isocomfort working area can reveal the operational cost according to working comfort, and provide more detailed information related to the workplace design.

The major difference from the past research is the shape of the working area. The contour of isocomfort working area reflecting the natural movement of the arm shows a shape of ellipsoid while the others are semicirclular or extended semi-circlular. In addition, the size of isocomfort working area is quite different for each hand while the two conventional working areas present the same dimension for both hands. It is due to a person's handedness. It seems reasonable that the shape of the comfortable working area for a left-handed person has the reverse pattern. The isocomfort working areas presented in Figure 3 shows the working comfort of $95^{\text {th }}$ percentile male for a lever at the slope of $15^{\circ}$.

The permission of the torso movement in this study generates a wider working area than the existing maximum working area which was determined under the assumption of upright posture. No restriction on the torso movement would be more reasonable to reflect real working conditions. The difference of the preferred locations between the controls implies that the comfortable working area should be differently applied to the design according to the type of controls. The comfortable working areas generated are especially useful in the case that a fine control is needed for prolonged hours. The result can be used as a basic guideline for instrument panel layout. A computerized panel design system is being implemented.

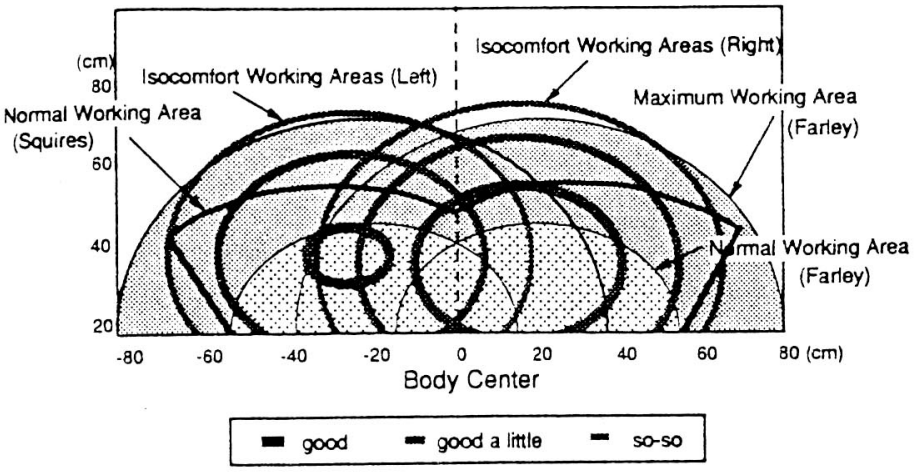

Figure 3. Comparison between isocomfort working areas and the existing working areas

\section{REFERENCES}

1) Farley, R. R. (1955). Some principles of methods and motion study as used in development work. General Motors Engineering Journal, 2, 20-25.

2) Jung, E. S., Park, S., and Han, S. H. (1995). Generation of Isocomfort Working Area based on the Psychophysical Evaluation. In Proceedings of the 39th Annual Meetings of Human Factors and Ergonomics Society, Santa Monica, CA, Human Factors and Ergonomic Society.

3) Myers, R. H. (1976), Response Surface Methodology, Blacksburg, Virginia, Virginia Polytechnic Institute and State University.

4) Squires, P. C. (1956). The shape of the normal working area. Report No. 275, U.S. Navy Department, Bureau of Medicine and Surgery, Medical Research Laboratories, New London, Connecticut. 